\title{
ARTICLE
}

\section{Comparison of activity among fluorescent lamps and light emitting diodes lamps exposed to secondary particles in accelerator rooms}

\author{
Akihiro Toyoda $^{\mathrm{a}^{*}}$, Hiroshi Matsumura ${ }^{\mathrm{a}}$, Kazuyoshi Masumoto ${ }^{\mathrm{a}}$, Shun Sekimoto ${ }^{\mathrm{b}}$, Hiroshi Yashima ${ }^{\mathrm{b}}$, Norihiro Matsuda $^{\mathrm{c}}$, \\ Yoshimi Kasugai ${ }^{c}$, Yukio Sakamoto ${ }^{c}$, Hiroshi Nakashima ${ }^{c}$, Gary Lauten $^{\mathrm{d}}$, Anthony Leveling ${ }^{\mathrm{d}}$, Nikolai Mokhov ${ }^{\mathrm{d}}$, \\ Kamran Vaziri ${ }^{\mathrm{d}}$, Koji Oishi ${ }^{\mathrm{e}}$, Makoto Kuroyanagi ${ }^{\mathrm{f}}$, Fumiyoshi Nobuhara ${ }^{\mathrm{f}}$ and Katsuhiko Takahashi ${ }^{\mathrm{g}}$ \\ ${ }^{a}$ High Energy Accelerator Research Organization, 1-1 Oho Tsukuba, Ibaraki 305-0801, Japan; ${ }^{b}$ Kyoto University Research Reactor \\ Institute, 2 Asashiro-Nishi, Kumatori-cho, Sennan-gun, Osaka 590-0494, Japan; ' Japan Atomic Energy Agency, 2-4 Shirane \\ Shirakata, Tokai-mura, Naka-gun, Ibaraki, 319-1195, Japan; ${ }^{d}$ Fermi National Accelerator Laboratory, Batavia, IL 60510-5011, \\ USA; ${ }^{e}$ Shimizu Corporation, 3-4-17 Etchujima, Koto-ku, Tokyo 135-8530, Japan; ${ }^{f}$ Tokyo Nuclear Services, 1-3-5 Taito, Taito-ku, \\ Tokyo 110-0016, Japan; ${ }^{g}$ Dokkyo Medical University, 880 Kitakobayashi, Mibu-machi, Shimotsuga-gun, Tochigi 321-0293, Japan
}

\begin{abstract}
Normal-type fluorescent lamps, three-wavelength-type fluorescent lamps, and LED lamps were exposed to secondary particles during machine operation in the four different high-energy accelerator rooms at the Fermi National Accelerator Laboratory (FNAL), USA, and Dokkyo Medical University, Japan. After the exposure of the lamps, we determined the activity produced in the lamps and examined the lighting characteristics of the lamps. In order to compare activity production among the same types of the lamps, total fourteen kinds of the lamps were used for the exposure experiments. The least activity after 25 days of the exposure was exhibited by the LED lamps. In examining the lighting characteristics of the lamps, however, it was found that the LED lamps could not be used at thermal and epithermal neutron fluence exceeding $9.7 \times 10^{10} \mathrm{~cm}^{-2}$. It was found that normal-type fluorescent lamps are superior to the three-wavelength-type fluorescent lamps from the point of view of activity production. We concluded that normal-type fluorescent lamps are the most suitable for lighting in high-energy accelerator rooms.
\end{abstract}

\section{Keywords: fluorescent lamp; LED lamp; radioactive waste; accelerator}

\section{Introduction}

Many straight-type fluorescent lamps are used for lighting in the accelerator rooms of high-energy accelerator facilities. In these accelerator rooms, activation occurs not only in the main components of the accelerators but also in all of the materials in the accelerator rooms [1]. The fluorescent lamps in the accelerator rooms are also activated by secondary particles. The life of a fluorescent lamp is typically a few years, and after use, the activated fluorescent lamps have to be treated as radioactive waste. As a result, a large amount of these activated lamps accumulates every year. Therefore, reduction of this radioactive waste is an important subject for waste management.

Although there are many kinds of commercial fluorescent lamps with different color temperatures, they can be categorized roughly into two groups according to phosphors. One is of the normal type, which emits white light from calcium halophosphate phosphors. The other is of the three-wavelength type, which emits light peaked at three different color wavelengths from rare-earth phosphors. The different phosphors in the

\footnotetext{
* Corresponding author. Email: akihiro.toyoda@kek.jp
}

fluorescent lamps can result in different radionuclide production and activity composition.

Recently, straight-tube-type light-emitting diode (LED) lamps have gained rapid popularity in Japan because of their long life and low power consumption. A socket for a fluorescent lamp can be used for an LED lamp as is. Thus if LED lamps can be used in accelerator facilities instead of the fluorescent lamps, it is expected that the volume of radioactive waste can be drastically reduced because of their long life. However, before replacing fluorescent lamps with LED lamps in accelerator rooms, we had to clarify the activity produced in the LED lamps and the durability of the LED lamps under radiation.

Therefore, in order to compare benefits in activity production and durability under radiation among the normal-type fluorescent lamp, three-wavelength-type fluorescent lamp, and LED lamp, various fluorescent and LED lamps were exposed to secondary particles during machine operation in the four different accelerator rooms. After the exposure of the lamps, we determined the activity produced in the lamps and examined the lighting characteristics of the lamps. Finally, from the results, we determined the best type of 
the lamp for high-energy accelerator rooms.

\section{Experimental procedures}

\subsection{Lamp samples for exposure}

The straight-tube normal-type fluorescent lamps, three-wavelength-type fluorescent lamps, and LED lamps exposed in this study are listed in Table 1. In order to compare radioactivity within the same type of fluorescent lamps, six normal-type and four three-wavelength-type fluorescent lamps operating at different temperatures were selected for the exposure experiments. Furthermore, four LED lamps made by different manufactures were also selected.

Table 1. Information of the light tubes for irradiation.

\begin{tabular}{cccc}
\hline Lamp type & Model name & $\begin{array}{c}\text { Color } \\
\text { temperature }\end{array}$ & Manufacture \\
\hline Fluorescent & F15T8/CW & $4100 \mathrm{~K}$ & General Electric \\
lamp & F15T8/KB & $3000 \mathrm{~K}$ & General Electric \\
(normal) & F15T8/PL/AQ & $3100 \mathrm{~K}$ & General Electric $^{*}$ \\
& FL10D & $6500 \mathrm{~K}$ & MEO $^{*}$ \\
& FL10N & $5000 \mathrm{~K}$ & MEO $^{*}$ \\
\hline Fluorescent & FL10EX-N TT & $5000 \mathrm{~K}$ & MEO $^{*}$ \\
lamp (three- & FL10EX-D TT & $6700 \mathrm{~K}$ & MEO $^{*}$ \\
wavelength) & FL10ENXF & $5800 \mathrm{~K}$ & MEO $^{*}$ \\
& FL10EDXF & $8000 \mathrm{~K}$ & MEO $^{*}$ \\
\hline LED lamp & LTL-10W & $4600 \mathrm{~K}$ & OnWave Corp. $^{*}$ \\
& LTL-10L & $3000 \mathrm{~K}$ & OnWave Corp. \\
& EH33-T78 & $5500-6000 \mathrm{~K}$ & e-halfCo., Ltd $^{*}$ \\
& MWT-10SMD-CL & $6500-7000 \mathrm{~K}$ & BLUE WING Inc. \\
\hline
\end{tabular}

*MITSSUBISHI ELECTRIC OSRAM Ltd.

The wattage, length, and outside diameter of the fluorescent lamps were, respectively, $15 \mathrm{~W}, 457.2 \mathrm{~mm}$, and $25.5 \mathrm{~mm}$ for lamps manufactured by General Electric and $10 \mathrm{~W}, 330 \mathrm{~mm}$, and $25.5 \mathrm{~mm}$ for those manufactured by Mitsubishi Electric Osram, Ltd.

The length and outside diameter of the LED lamps were $330 \mathrm{~mm}$ and $30 \mathrm{~mm}$, respectively. Many LEDs were lined doubly or triply on a printed circuit board in acrylic or polycarbonate tubes. These could be installed in a socket for a straight $10-\mathrm{W}$ fluorescent lamp.

\subsection{Exposure of the lamp samples}

The lamp samples were exposed to secondary particles in four different accelerator rooms in Fermi National Accelerator Laboratory (FNAL), USA, and Dokkyo Medical University, Japan. The experimental conditions of the exposure experiments are listed in Table 2. Acceleration beams in FNAL and Dokkyo Medical University were $120-\mathrm{GeV}$ protons and $18-\mathrm{MeV}$ protons, respectively. The lamps were exposed for terms long enough to produce sufficient activity in the lamps for $\gamma$-ray spectrometry.
Table 2. Information of the exposure experiments.

\begin{tabular}{cccccc}
\hline $\begin{array}{c}\text { Exposure } \\
\text { name }\end{array}$ & $\begin{array}{c}\text { Exposure } \\
\text { place }\end{array}$ & Facility & Beam & $\begin{array}{c}\text { Total } \\
\text { proton }\end{array}$ & $\begin{array}{c}\text { Machine } \\
\text { operation time } \\
\text { (term })\end{array}$ \\
\hline Exp1 & $\begin{array}{c}\text { NuMI Hadron } \\
\text { absorber }\end{array}$ & FNAL, USA & $\begin{array}{c}120-\mathrm{GeV} \\
\text { proton }\end{array}$ & $6.1 \times 10^{18}$ & $\begin{array}{c}4.8 \text { days } \\
(5 \text { days })\end{array}$ \\
Exp2 & $\begin{array}{c}\text { NuMI Hadron } \\
\text { absorber }\end{array}$ & FNAL,USA & $\begin{array}{c}120-\mathrm{GeV} \\
\text { proton }\end{array}$ & $1.5 \times 10^{19}$ & $\begin{array}{c}12.9 \text { days } \\
(13 \text { days })\end{array}$ \\
Exp3 & $\begin{array}{c}\text { NuMI } \\
\text { Alcove-1 }\end{array}$ & FNAL, USA & $\begin{array}{c}120-\mathrm{GeV} \\
\text { proton }\end{array}$ & $1.6 \times 10^{20}$ & $\begin{array}{c}112.9 \text { days } \\
(132 \text { days })\end{array}$ \\
Exp4 & $\begin{array}{c}\text { Main } \\
\text { injector }\end{array}$ & FNAL, USA & $\begin{array}{c}120-\mathrm{GeV} \\
\text { proton }\end{array}$ & $1.5 \times 10^{19}$ & $\begin{array}{c}12.9 \text { days } \\
(13 \text { days })\end{array}$ \\
Exp5 & $\begin{array}{c}\text { Cyclotron } \\
\text { room }\end{array}$ & $\begin{array}{c}\text { Dokkyo Medical } \\
\text { Univ., Japan }\end{array}$ & $\begin{array}{c}18-\mathrm{MeV} \\
\text { proton }\end{array}$ & $3.5 \times 10^{19}$ & $\begin{array}{c}1.2 \text { days } \\
(32 \text { days })\end{array}$ \\
\hline
\end{tabular}

In Exp1 and Exp2, the lamps were placed outside the Hadron absorber, in the NuMI Hadron absorber hall in FNAL (NuMI Hadron absorber). Here, two Au foils (15 $\times 15 \times 0.02 \mathrm{~mm}^{3}$ ), one of which was covered by a 0.5-mm-thick cadmium plate, were also placed alongside the lamps to estimate thermal and epithermal neutron fluxes. The estimated fluxes of thermal and epithermal neutrons were $(1.9 \pm 0.2) \times 10^{7} \mathrm{~cm}^{-2} \mathrm{~s}^{-1}$ and $(6.5 \pm 0.3) \times 10^{5} \mathrm{~cm}^{-2} \mathrm{~s}^{-1}$, respectively.

In the Exp3, the lamps were exposed in Alcove-1 of the NuMI beamline in FNAL (NuMI Alcove-1). NuMI Alcove-1 is located downstream from the NuMI hadron absorber on the beam axis. High-energy hadrons and muons might pass through this exposure location.

In Exp4, the lamps were placed on the wall near a $120-\mathrm{GeV}$-proton beam collimator in the main injector tunnel (Main injector). During the exposure term, the main injector was operating regularly.

In Exp5, the lamps were placed near a wall at $1.5-\mathrm{m}$ distance from a target box in a cyclotron room in Dokkyo Medical University (Cyclotron room). The cyclotron was operated to produce isotopes for positron emission tomography (PET) for $1 \mathrm{~h}$ each day.

\subsection{Gamma-ray spectrometry}

The radioactivity produced in the lamps was determined by $\gamma$-ray spectrometry using Ge detectors. The $\gamma$-ray measurements were performed typically after 3 weeks and again after 5 months of exposure. During $\gamma$-ray measurement, the Ge-detector head was shielded by lead blocks with a 10 -cm-wide or 15-cm-wide mouth open in the sample direction. The lamp sample was installed on a vertical plane for the head axis behind the opened mouth in order to detect the $\gamma$-rays from only the main body of the lamp by the Ge detector. The $\gamma$-rays from the metallic electrodes on both ends of the lamps were not measured.

Two of the Ge detectors were previously calibrated by Canberra [2]. Others were not calibrated. The efficiencies of the previously calibrated detectors were determined using Canberra's ISOCS/LabSOCS [3] software. In the case of the fluorescent lamp, the $\gamma$-ray detection efficiency was obtained using the software by assuming the fluorescent lamp to be a glass cylinder. In case of the LED lamps, the main component is the printed circuit board. The $\gamma$-ray detection efficiency of 
the printed circuit board was obtained using the software by assuming the board to be a polycarbonate plate. The efficiency of the uncalibrated detectors was determined by comparing count rates for the same sample obtained by the calibrated and uncalibrated detectors. Finally, we obtained the activity from the entire tube.

\subsection{Lighting test of the exposed lamps}

After exposure of the lamps, the lighting characteristics of the lamps were assessed by visual observation. Note, however, that the lighting characteristics of the fluorescent lamps manufactured by General Electric exposed in Exp1 were not examined.

\section{Results and discussion}

\subsection{Activities produced in the exposed lamps}

Activities of radionuclides 25 days after the end of the exposure are shown in Figures 1 (a-1) and (a-2) for the NuMI Hadron absorber, (b) for NuMI Alcove-1, (c) for the Main injector, and (d) for the Cyclotron room. These activities were calculated using the activity measured 27 days after exposure for Figure (a-1), 300 days after exposure for (a-2), 27 days after exposure for (b), 8 hours after exposure for (c), and 85 days after exposure for (d). Counting errors of activity measurement of the samples was generally within 25\%. Although short half-life nuclides such as ${ }^{24} \mathrm{Na}$ were determined from early measurements, radionuclides with half-lives shorter than 4 days are not shown in Figure 1, because these radionuclides are not important for radioactive waste management.

Detected radionuclides were different among the normal-type fluorescent lamps, three-wavelength-type fluorescent lamps, and LED lamps. In both the normal-type and three-wavelengths-type fluorescent lamps, similar activity of ${ }^{124} \mathrm{Sb}$ (half-life: 60.20 days) was strongly induced by thermal neutron capture reaction of ${ }^{123} \mathrm{Sb}$. In the three-wavelength-type fluorescent lamps, the activities of ${ }^{152} \mathrm{Eu}$ (half-life: 13.542 years) and ${ }^{160} \mathrm{~Tb}$ (half-life: 72.3 days) were determined to be higher than the activity of ${ }^{124} \mathrm{Sb}$. Because rare-earth phosphors are used in the three-wavelength-type fluorescent lamps, ${ }^{152} \mathrm{Eu}$ and ${ }^{160} \mathrm{~Tb}$ were produced by neutron capture reactions of ${ }^{151} \mathrm{Eu}$ and ${ }^{159} \mathrm{~Tb}$, respectively. The production of the ${ }^{152} \mathrm{Eu}$ and ${ }^{160} \mathrm{~Tb}$ activities was independent of light temperature. High activity of the long half-life ${ }^{152} \mathrm{Eu}$ is detrimental to radioactive waste reduction because long storage is required. In a high-energy particle field such as at NuMI Alcove-1, activities of ${ }^{7} \mathrm{Be}$ and ${ }^{22} \mathrm{Na}$, which could be produced by spallation of oxygen and silicon nuclei, were added to the list of activities, as shown in Figure 1 (c). These results obviously indicate that the normal type fluorescent lamps are superior to the three-wavelength-type fluorescent lamps from the point of view of activity production.

The radionuclides detected in the LED lamps were different from those in the fluorescent lamps. The radionuclides ${ }^{110 \mathrm{~m}} \mathrm{Ag},{ }^{65} \mathrm{Zn},{ }^{58} \mathrm{Co}$, and ${ }^{182} \mathrm{Ta}$ were attributed to the peculiarities of the products in the LED lamps. The half-lives of these nuclides are shorter than 1 year. In a high-energy particle field such as that at NuMI Alcove-1, activities of ${ }^{7} \mathrm{Be}$ and ${ }^{22} \mathrm{Na}$, which could be produced by spallation, were added to the list of activities just as for the fluorescent lamps. The activity produced in the LED lamps was much lower than that in the fluorescent lamps. From the point of view of activity production, we found that LED lamps are superior to fluorescent lamps.

Short half-life nuclides are related to personnel radiation exposure from the lamps. In Exp1, early measurement was carried out for the normal-type fluorescent lamps and LED lamps, $6 \mathrm{~h}$ after exposure. The main radionuclides were different among the lamps. In the normal type fluorescent lamps manufactured by General Electric, $0.97-1.26 \times 10^{6} \mathrm{~Bq}$ of ${ }^{24} \mathrm{Na}$ (half-life: $14.9590 \mathrm{~h}$ ) were determined as the source of most of the activity. It is assumed that ${ }^{24} \mathrm{Na}$ was produced by thermal neutron capture of ${ }^{23} \mathrm{Na}$ in the glass. In the LED lamps, $2.5-4.5 \times 10^{6} \mathrm{~Bq}$ of ${ }^{64} \mathrm{Cu}$ (half-life: $12.700 \mathrm{~h}$ ) and $1.9-5.1 \times 10^{5} \mathrm{~Bq}$ of ${ }^{82} \mathrm{Br}$ (half-life: $35.30 \mathrm{~h}$ ) were determined as the main sources of activity. Those might have been produced from materials in the printed circuit board. The contribution of ${ }^{82} \mathrm{Br}$ to the dose rate was comparable to or greater than that of ${ }^{64} \mathrm{Cu}$.

\subsection{Lighting test of the exposed lamps}

In the case of the fluorescent lamps, all of the exposed lamps could be illuminated completely except for FL10D in Exp4. However, in the case of the LED lamps, radiation damage was observed in lighting tests for most of the exposed lamps, and only LTL10W and LTL10L in Exp1 and EH33-T78 in Exp5 could be illuminated. EH33-T78 in Exp3 failed, and other lamps were blinking. These results indicate that the fluorescent lamps have high resistance to radiation damage, while the LED lamps are not very resistant. Relative neutron fluences among the exposure experiments were estimated roughly using ${ }^{152} \mathrm{Eu}$ activity produced in the same three-wavelength-type fluorescent lamps. According to the ${ }^{152} \mathrm{Eu}$ activity, the neutron fluence in Exp5 was the least among the exposure experiments. In pre-investigation of the neutron flux using $\mathrm{Au}$ and $\mathrm{Cd}$-covered $\mathrm{Au}$ foils, the thermal and epithermal neutron fluxes were estimated to be $(8.9 \pm 1.0) \times 10^{5}$ and $(3.9 \pm 0.2) \times 10^{4} \mathrm{~cm}^{-2} \mathrm{~s}^{-1}$, respectively, at the exposure location in the Cyclotron room. Therefore, it was found that the LED lamps could not be used at thermal and epithermal neutron fluences exceeding $(9.7 \pm 1.1) \times 10^{10}$ $\mathrm{cm}^{-2}$.

\section{Conclusion}

In this study, normal-type fluorescent lamps, three-wavelength-type fluorescent lamps, and LED lamps were exposed to secondary particles during 


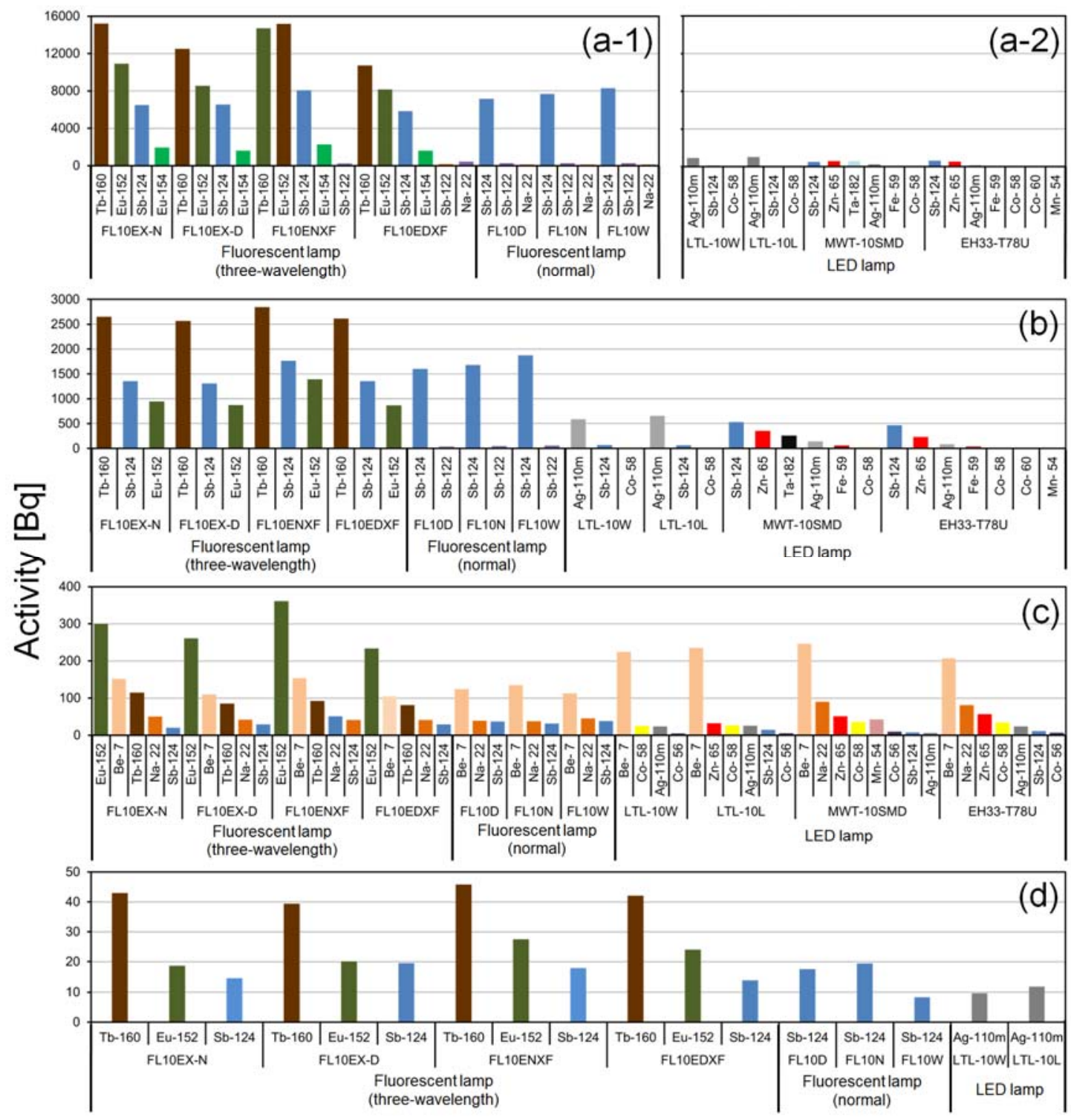

Figure 1. Activities of radionuclides 25 days after the exposure. The values were calculated from the activities measured in the exposed lamps. (a): NuMI Hadron absorber, (b): Main injector, (c): NuMI Alcove-1, and (d): Cyclotron room.

machine operation in four different high-energy accelerator rooms. The induced activities 25 days after the end of exposure were lowest for the LED lamps and highest for the three-wavelength-type fluorescent lamps. In examining the lighting characteristics of the lamps, however, it was found that the LED lamps could not be used at thermal and epithermal neutron fluences exceeding $9.7 \times 10^{10} \mathrm{~cm}^{-2}$. We concluded that normal-type fluorescent lamps are the most suitable illumination lamp for high-energy accelerator rooms.

\section{Acknowledgements}

This work was supported by a grant-in-aid from the Ministry of Education, Science and Culture (KAKENHI 19360432 and 21360473) in Japan. Fermilab is a US Department of Energy Laboratory operated under Contract DE-AC02-07CH11359 by the Fermi Research Alliance, LLC.

\section{References}

[1] H. W. Patterson and R. H. Thomas, Accelerator Health Physics, Academic Press, Inc., New York, (1973).

[2] R. Venkataraman, F. Bronson, V. Atrashkevich, M. Field and B. M. Young, Improved detector response characterization method in ISOCS and LabSOCS, $J$. Radioanal. Nucl. Chem. 264 (2005), pp. 213-219.

[3] F. L. Bronson, Validation of the accuracy of the LabSOCS software for mathematical efficiency calibration of $\mathrm{Ge}$ detectors for typical laboratory samples, J. Radioanal. Nucl. Chem. 255 (2003), pp. $137-141$. 